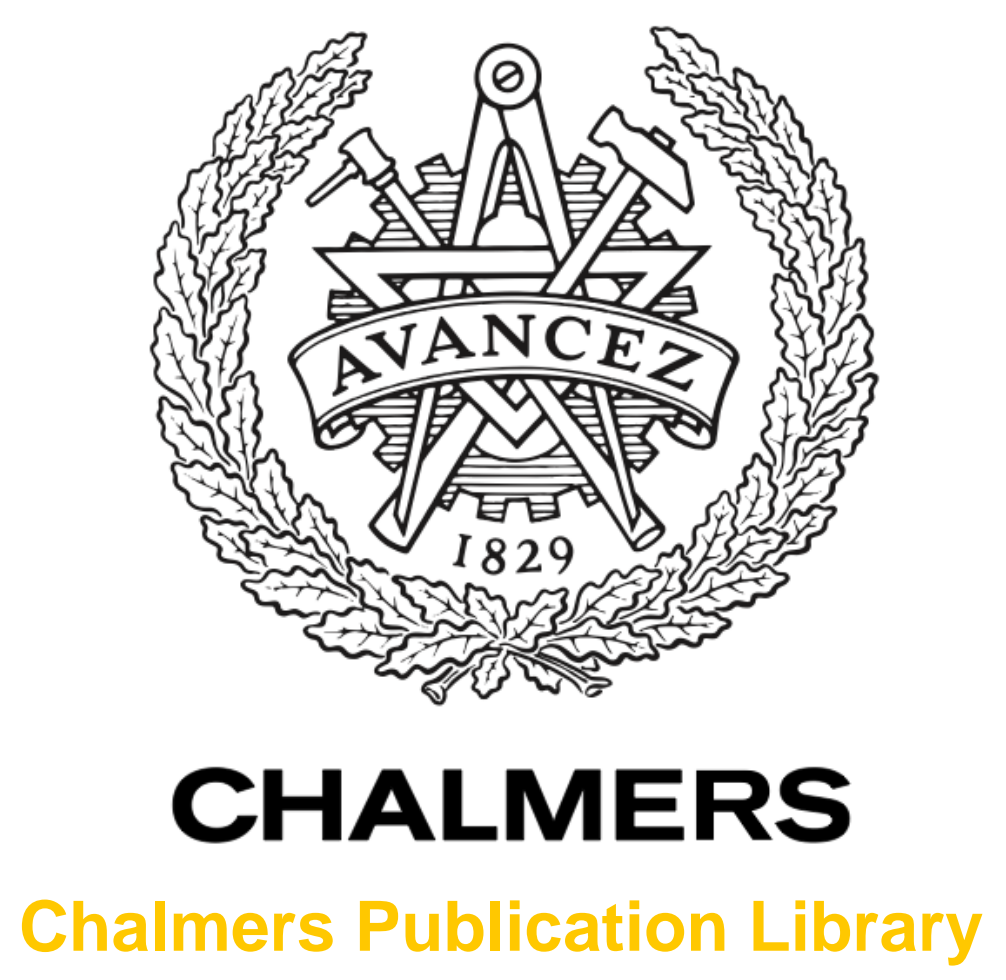

On the Trade-off Between Accuracy and Delay in Cooperative UWB Navigation

This document has been downloaded from Chalmers Publication Library (CPL). It is the author's version of a work that was accepted for publication in:

Proceeedings of 2013 IEEE Wireless Communications and Networking Conference (WCNC)

Citation for the published paper:

Garcia, G. ; Muppirisetty, L. ; Wymeersch, H. (2013) "On the Trade-off Between Accuracy and Delay in Cooperative UWB Navigation". Proceeedings of 2013 IEEE Wireless

Communications and Networking Conference (WCNC)

Downloaded from: http://publications.lib.chalmers.se/publication/175711

Notice: Changes introduced as a result of publishing processes such as copy-editing and formatting may not be reflected in this document. For a definitive version of this work, please refer to the published source. Please note that access to the published version might require a subscription.

Chalmers Publication Library (CPL) offers the possibility of retrieving research publications produced at Chalmers University of Technology. It covers all types of publications: articles, dissertations, licentiate theses, masters theses, conference papers, reports etc. Since 2006 it is the official tool for Chalmers official publication statistics. To ensure that Chalmers research results are disseminated as widely as possible, an Open Access Policy has been adopted.

The CPL service is administrated and maintained by Chalmers Library. 


\title{
On the Trade-off Between Accuracy and Delay in Cooperative UWB Navigation
}

\author{
Gabriel E. Garcia, L. Srikar Muppirisetty, and Henk Wymeersch \\ Department of Signals and Systems, Chalmers University of Technology, Gothenburg, Sweden \\ E-mail: \{ggarcia,srikar.muppirisetty,henkw\} @ chalmers.se
}

\begin{abstract}
In ultra-wide bandwidth (UWB) cooperative navigation, nodes estimate their position by means of shared information. Such sharing has a direct impact on the position accuracy and medium access control (MAC) delay, which needs to be considered when designing UWB navigation systems. We investigate the interplay between UWB position accuracy and MAC delay for cooperative scenarios. We quantify this relation through fundamental lower bounds on position accuracy and MAC delay for arbitrary finite networks. Results show that the traditional ways to increase accuracy (e.g., increasing the number of anchors or the transmission power) as well as inter-node cooperation may lead to large MAC delays. We evaluate one method to mitigate these delays.
\end{abstract}

\section{INTRODUCTION}

Absolute and relative position information has become essential in the development of location-aware applications. Such applications pervade the commercial, public, and military domains [1]. Ultra-wide bandwidth (UWB) communication and ranging has shown to be a promising technology to solve the navigation problem in scenarios where GPS-aided implementations are not viable. UWB radios employ a pulsebased approach with absolute bandwidths of more than 500 $\mathrm{MHz}$ [2]. This characteristic offers several communication advantages, such as mitigation of small-scale fading and robustness against interference [3]. Furthermore, the precision of range measurements, using a two-way time-of-arrival (TWTOA) procedure, is proportional to the employed bandwidth, making UWB an attractive technology for localization and navigation purposes [4].

A large research effort has focused on developing ways to improve navigation accuracy. One research track involves cooperation among nodes to distribute and share information over the network, which has been shown to improve position accuracy significantly [5], [6]. Position accuracy for cooperative wideband systems, such as UWB, has been studied in [7], [8]. However, these works do not consider the influence on the medium access control (MAC) within practical implementations.

The impact and design of MAC, constrained to the IEEE 802.15.4a standard has been studied in [9], [10]. In [9], the authors propose enhancements to the 802.15.4a standard using a time division multiple access (TDMA) approach for clique networks. Further improvements to [9] are proposed in [10], where the authors reduce the MAC delay, but do not consider the impact on position accuracy. An evaluation of different TDMA-based prioritization strategies is studied in [11]. In [12], an IEEE 802.11.b MAC is considered and based on a cooperative model the interplay between MAC delay and position accuracy is explored. However, regarding throughput, this MAC is inefficient and thus produces excessively pessimistic delay estimates. Distributed scheduling for cooperative localization is investigated in [13], while in [14], a distributed MAC protocol was suggested, suitable for cooperative UWB navigation in clique networks. Nevertheless, they do not consider the trade-off between accuracy and MAC delay. Finally, a MAC design for cooperative localization networks is studied in [15]. However, the authors only consider the specific analysis and design of the MAC protocol.

In this paper, we extend our work from [16], and derive lower bounds on UWB position accuracy and MAC delay for noncooperative and cooperative networks based on a spatial time division multiple access (STDMA) approach. This allows an understanding of the fundamental trade-off between accuracy and MAC delay with immediate application to the design of UWB navigation systems. Results show large delays are incurred when cooperation is performed indiscriminately. A selective ranging method to reduce MAC delay at the expense of position accuracy is evaluated.

\section{SySTEM MODEL}

\section{A. UWB Navigation}

We consider a wireless network consisting of $N$ mobile agent nodes and $M$ static anchors nodes, collected in the sets $\mathcal{S}_{\text {agents }}$ and $\mathcal{S}_{\text {anchors }}$, respectively. Agents move in discrete time slots of duration $T$. The two-dimensional position of node $i$ at time slot $t$ is denoted by $\mathbf{x}_{i}^{(t)}$. The measurements made by agent $i$ at time slot $t$ are denoted by $\mathbf{z}_{i}^{(t)}$. We assume the following discrete-time model for the agents [17]:

$$
\begin{aligned}
& \mathbf{x}_{i}^{(t)}=f_{\text {state }}\left(\mathbf{x}_{i}^{(t-1)}\right)+\mathbf{v}_{i}^{(t)} \\
& \mathbf{z}_{i}^{(t)}=f_{\text {meas }}\left(\mathbf{x}_{i}^{(t)}\right)+\mathbf{w}_{i}^{(t)}
\end{aligned}
$$

where the noise terms $\mathbf{v}_{i}^{(t)}$ and $\mathbf{w}_{i}^{(t)}$ capture the randomness and errors within the mobility and measurement model, respectively. The position of agent $i$ is estimated by recursively predicting an a priori distribution, $p\left(\mathbf{x}_{i}^{(t)} \mid \mathbf{z}_{i}^{(1: t-1)}\right)$, and then correcting to an a posteriori distribution $p\left(\mathbf{x}_{i}^{(t)} \mid \mathbf{z}_{i}^{(1: t)}\right)$ using the available measurements, before moving on to the next time slot. Evidently, faster mobility requires the usage of shorter slots. 
The time slot duration $T$ can be broken down into a measurement time $T_{\text {meas }}$ and a computation time $T_{\text {comp }}$. The measurement time consists of the time required by the agents to collect all necessary measurements (ranging information) while the computation time relates to the time it takes to calculate the posterior distribution. It is important to note that UWB is used for the measurement phase. Delays incurred when agents send information to each other (distributed approach) or to a fusion center (centralized approach), once all data is collected, can be implemented using an alternative technology, and are therefore not included in our analysis. Thus, our objective is to quantify how adding more anchors or agents, increasing the communication range, and the use of cooperation among agents affects the position accuracy and the MAC delay.

Accuracy and MAC delay are evaluated using the position error bound (PEB) [7], [8], [18] and the minimal $T_{\text {meas }}$ required to collect all information by the agents, respectively. We assume a given a priori distribution for every agents' position so that we can focus on a single time slot within the navigation procedure. For notational convenience, the superscript $t$ will be dropped.

\section{B. Network Model}

Adopting the model from [19], nodes $i$ and $j$ can communicate with probability $P_{i j}=\exp \left(-\left\|\mathbf{x}_{i}-\mathbf{x}_{j}\right\|^{2} /\left(2 R^{2}\right)\right)$, where $R$ is the nominal communication range in meters. The set of neighbors of a node $i$ is denoted by $\mathcal{N}_{i}=\{j \neq i: i$ and $j$ can communicate $\}$. Node $i$ can perform TW-TOA ranging with any node in $\mathcal{N}_{i}$.

Two TW-TOA transactions can only be performed simultaneously if they do not interfere with each other. As 802.15.4a radios use a common preamble, similar to off-the-shelf UWB radios [20], we cannot rely on time-hopping to deal with interference, and traditional protocols such as ALOHA or slotted ALOHA have poor efficiency in terms of successful number of transactions [21]. Hence, we consider a STDMA MAC as in [9]-[11]. This implies, for example, that when four radios involved in two TW-TOA transactions are adjacent, then the two transactions must be scheduled in distinct TDMA slots.

\section{Measurement Model}

We consider TW-TOA measurements [2], whereby a node $i$ sends a request to a node $j$, which responds back with an acknowledgement. Both nodes $i$ and $j$ estimate the TOA for the request and acknowledgment, respectively. Node $i$ uses the round-trip delay between nodes $i$ and $j$ to calculate an estimate of their distance. It is important to note that a TDMA slot is needed for a TW-TOA transaction, including both the request and the acknowledgement. The TW-TOA measurement between node $i$ and node $j$ is given by:

$$
z_{i j}=d_{i j}+\frac{n_{i j}}{2}+\frac{n_{j i}}{2}
$$

where $d_{i j}=\left\|\mathbf{x}_{i}-\mathbf{x}_{j}\right\|, n_{i j}$ is the TOA error of the request from node $i$ to node $j$ and $n_{j i}$ is the TOA error from the acknowledgement from node $j$ to node $i$. The errors are modeled as zero-mean Gaussian random variables: $n_{i j} \sim \mathcal{N}\left(0, \sigma_{i j}^{2}\right)$ and $n_{j i} \sim \mathcal{N}\left(0, \sigma_{j i}^{2}\right)$, independent of each other.

\section{LOWER BOUND ON POSITION ACCURACY}

In this section, we will review the position error bound (PEB), when performing TW-TOA within the network topology described in Section II, for noncooperative and cooperative scenarios [7], [8], [18]. We collect the positions of all the agents in a vector $\mathbf{p}=\left[\mathbf{x}_{1}^{\mathrm{T}} \mathbf{x}_{2}^{\mathrm{T}} \cdots \mathbf{x}_{N}^{\mathrm{T}}\right]^{\mathrm{T}}$. Since we are focusing on a single time slot, every agent has an a priori distribution, which for simplicity is modeled as a symmetric Gaussian distribution with mean $\mathbf{m}_{\text {prior }, i}$ and variance $\sigma_{\text {prior }, i}^{2}$ per dimension.

With a slight abuse of notation, and introducing the set $\mathcal{S}_{i} \subseteq \mathcal{N}_{i}$ consisting of selected neighbors with which node $i$ performs TW-TOA ranging, the measurement vector for both the noncooperative or cooperative cases can be constructed as:

$$
\mathbf{z}=\left\{z_{i j} \mid i \in \mathcal{S}_{\text {agents }}, j \in \mathcal{S}_{i}\right\},
$$

in which $z_{i j}$ contains the TW-TOA estimate initiated between agent $i$ with respect to node $j$. Note that in non-cooperative scenarios, $\mathcal{S}_{i} \subseteq \mathcal{N}_{i} \cap \mathcal{S}_{\text {anchors }}$, while in a cooperative scenario, $\mathcal{S}_{i} \subseteq \mathcal{N}_{i}$.

Due to (3), $\mathbf{z}$ conditioned on $\mathbf{p}$ is a Gaussian random variable with mean $\mathbf{d}$, constructed from (3) and in the same way as $\mathbf{z}$, and covariance matrix $\boldsymbol{\Sigma}$. As a result of the independence of the TW-TOA measurements, the covariance matrix

$$
\boldsymbol{\Sigma}=\mathbb{E}\left\{(\mathbf{z}-\mathbf{d})(\mathbf{z}-\mathbf{d})^{\mathrm{T}}\right\}
$$

is a diagonal matrix, where the diagonal element corresponding to measurement $z_{i j}$ is given by

$$
\mathbb{E}\left\{\left(z_{i j}-d_{i j}\right)^{2}\right\}=\frac{\sigma_{i j}^{2}+\sigma_{j i}^{2}}{4} .
$$

Finally, the PEB is defined as $\mathcal{P}=\sqrt{\operatorname{tr}\left\{\mathbf{J}^{-1}\right\} / N}$, where $\mathbf{J}$ is the Bayesian Fisher information matrix and is computed as [22]:

$$
\begin{aligned}
\mathbf{J} & =-\mathbb{E}_{\mathbf{p}, \mathbf{z}}\left\{\nabla_{\mathbf{p}} \nabla_{\mathbf{p}}^{\mathrm{T}} \log p(\mathbf{z}, \mathbf{p})\right\} \\
& =-\mathbb{E}_{\mathbf{p}, \mathbf{z}}\left\{\nabla_{\mathbf{p}} \nabla_{\mathbf{p}}^{\mathrm{T}} \log p(\mathbf{z} \mid \mathbf{p})\right\}-\mathbb{E}_{\mathbf{p}}\left\{\nabla_{\mathbf{p}} \nabla_{\mathbf{p}}^{\mathrm{T}} \log p(\mathbf{p})\right\} \\
& =-\mathbb{E}_{\mathbf{p}}\left\{\nabla_{\mathbf{p}} \mathbf{d}^{\mathrm{T}} \boldsymbol{\Sigma}^{-1} \nabla_{\mathbf{p}}^{\mathrm{T}} \mathbf{d}\right\}+\mathbf{D},
\end{aligned}
$$

where $\mathbf{D}$ denotes the $2 N \times 2 N$ diagonal matrix

$$
\mathbf{D}=\operatorname{diag}\left[\frac{1}{\sigma_{\text {prior }, 1}^{2}}, \frac{1}{\sigma_{\text {prior }, 1}^{2}}, \ldots, \frac{1}{\sigma_{\text {prior }, N}^{2}}, \frac{1}{\sigma_{\text {prior }, N}^{2}}\right],
$$

and $\nabla_{\mathbf{p}}$ stands for the derivative with respect to $\mathbf{p}$. Here, the entries in the matrix $\nabla_{\mathbf{p}} \mathbf{d}^{\mathrm{T}}$ can easily be calculated since

$$
\frac{\partial d_{i j}}{\partial \mathbf{x}_{i}}=\frac{\mathbf{x}_{i}-\mathbf{x}_{j}}{d_{i j}}
$$

Through the use of Monte Carlo integration, the expectation over $\mathbf{p}$ in (7) can be computed. Note that evaluation of the PEB is computationally easy, since $\boldsymbol{\Sigma}$ is diagonal and $\nabla_{\mathbf{p}} \mathbf{d}^{\mathrm{T}}$ is sparse. 


\section{Bounds ON Minimum MAC DELAY}

For a ranging transaction to be successful, the link should be free from primary interference (i.e., a node cannot transmit and receive at the same time) and secondary interference (i.e., a node cannot receive multiple transmissions at the same time) [23]. Note that this applies to both nodes in the link, since ranging transactions involve both nodes and thus links are undirected. In the noncooperative localization scenario, a link (always an agent-to-anchor link) is scheduled only once. In the cooperative localization scenario, some agent-to-agent links may be scheduled twice, i.e., when $i, j \in \mathcal{S}_{\text {agents }}$ such that $i \in \mathcal{S}_{j}$ and $j \in \mathcal{S}_{i}$. We will first show how to construct the communication graph for our navigation network and then derive bounds on minimum MAC delay.

\section{A. Construction of Communication Graph}

Once the links are realized (see Section II.B), the network can be described by a communication graph $G=(V, E)$ consisting of a set $V$ of vertices (nodes) and a set $E$ representing the edges (links) amongst the vertices. Depending on the sets $\mathcal{S}_{i}$, recall that some links are scheduled once, some twice, and some not at all. The graph $G$ can be extended to a new graph $G^{\prime}=\left(V^{\prime}, E^{\prime}\right)$ to accommodate these different number of scheduled links. Let us assume that the link $(u, v) \in E$ requires two time slots. The construction of $G^{\prime}$ is as follows: create a new vertex $u^{\prime}$, serving as a copy of $u$. Create edges $\left(u^{\prime}, w\right)$, for all $w \in \mathcal{N}_{u}$, as well as an edge $\left(u, u^{\prime}\right)$. Note that the degree of nodes $u$ and $v$ and total number of nodes is increased by 1 in the extended graph $G^{\prime}$. This procedure is repeated for all links that require two slots.

Upon completion of the procedure we find a graph $G^{\prime}$, with $V^{\prime} \supseteq V$ and $E^{\prime} \supseteq E$. Let $W$ be the number of links in $G$ that need two time slots, then the number of nodes in the corresponding extended graph $G^{\prime}$ is increased by $W$. Fig. 1 illustrates the construction of the extended graph, where the link $(1,4)$ requires two time slots.

Let $\mathbf{A}$ be the symmetric adjacency matrix of graph $G^{\prime}$ with $A_{i j}=1, \forall(i, j) \in E^{\prime}$. We further introduce $\mathbf{S}$, the symmetric scheduling matrix, with $S_{i j}=1$ if the link $(i, j)$ is to be scheduled for TW-TOA, and $S_{i j}=0$ otherwise. Note that $S_{i j}=1 \Rightarrow A_{i j}=1$, and that by construction, links in $G^{\prime}$ are scheduled at most once.

\section{B. Upper and Lower Bounds on Minimum MAC Delay}

Finding the exact number of time slots to schedule the links in $G^{\prime}$ is an NP-complete problem [24]. This problem can be avoided by considering special networks [10] or resorting to computer simulations [9], [11], [12]. Instead we focus on finding lower $(\Upsilon)$ and upper $(\Omega)$ bounds on the total number of TDMA time slots to schedule the links from $\mathbf{S}$, given the adjacency matrix $\mathbf{A}$. The procedure below finds such bound with complexity $\mathcal{O}((N+M+W) \Lambda)$, where $\Lambda$ is the maximum A-degree. ${ }^{1}$

\footnotetext{
${ }^{1}$ The A-degree of node $i$ is the sum of the entries of the $i$-th row in $\mathbf{A}$
}

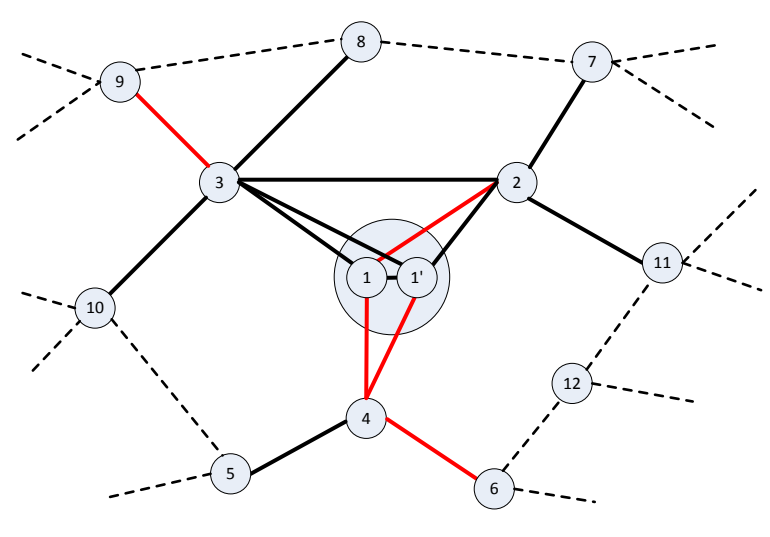

Figure 1. Subgraph (shown in thick lines) associated with node 1 within the larger graph $G^{\prime}$. For this subgraph, links $(1,2),(4,6)$ and $(3,9)$ require one time slot and the link $(1,4)$ requires two time slots. These links are marked red. The rest of the links (e.g., link $(3,2))$ are not scheduled. In this case, node 1 is split as nodes 1 and $1^{\prime}$. The neighbors of node 1 are nodes $2,3,4$ and $1^{\prime}$. So node $1^{\prime}$ is now connected to nodes $1,2,3$ and 4 .

Upper bounds: A trivial upper bound is the total number of links to be scheduled in the network, $\Omega_{1}=\left(\mathbf{1}^{\mathrm{T}} \mathbf{S} \mathbf{1}\right) / 2$, where $\mathbf{1}$ is a vector of ones. In this case interference is avoided by assigning time slots to links in TDMA fashion. A different upper bound was conjectured by Erdős-Nešetřil [25] and is given by $\Omega_{2}=\frac{5}{4} \Delta^{2}$ for even $\Delta$ and $\Omega_{2}=\frac{1}{4}\left(5 \Delta^{2}-2 \Delta+1\right)$ for odd $\Delta$, where $\Delta$ is the maximum $\mathbf{S}$-degree. ${ }^{2}$ The upper bound can furthermore be tightened by choosing the minimum of $\Omega_{1}$ and $\Omega_{2}$. Hence, our final upper bound is $\Omega=\min \left(\Omega_{1}, \Omega_{2}\right)$.

Lower bounds: A trivial, but very loose lower bound is $\Upsilon_{1}=\Delta$, since every link in $\mathbf{S}$ related to one node must be scheduled in a distinct TDMA slot. In other words each neighbor of the node is assigned a different TDMA slot. We also present a constructive lower bound, by considering simple subgraphs of the graph $G^{\prime}$ and finding a lower bound on the number of TDMA time slots for that subgraph (or corresponding subnetwork). The maximum of the lower bounds of these subnetworks gives a lower bound, $\Upsilon_{2} \geq \Upsilon_{1}$ for the entire network. Note that for clique networks, $\Upsilon_{2}=\Omega$.

The constructive lower bound for a subnetwork is obtained as follows: consider a node $i$, one of its neighbors $j$, as well as any common neighbors, $\mathcal{N}_{i} \cap \mathcal{N}_{j}$, for example $(i, j)=(1,4)$, $\mathcal{N}_{1} \cap \mathcal{N}_{4}=1^{\prime}$. The nodes/vertices adjacent to $i, j$, and $\mathcal{N}_{i} \cap \mathcal{N}_{j}$ are in this case $\left(1,1^{\prime}, 2,3,4,5,6\right)$. In order to schedule the links incident to those nodes, we need $\Delta_{1}+\left(\left(\Delta_{4}-1\right)+\right.$ $\left.\Delta_{1^{\prime}}\right)-1=4$ TDMA time slots. Here $\Delta_{i}$ is the $\mathbf{S}$-degree of node $i$ and the last term accounts for any common edges that would be assigned two slots. This procedure is repeated for all neighbors $j$ and the maximum number of TDMA time slots is retained.

An algorithm that formalizes this reasoning is given in Algorithm 1, with the following notations: $\mathbf{a}_{i}$ and $\mathbf{s}_{i}$ are the $i$-th column of the adjacency and scheduling matrix, respectively; $\mathbf{e}_{j}$ is a vector of all zeros except for a 1 at the $j$ th location; $\boldsymbol{\Delta}=\left[\Delta_{1}, \Delta_{2}, \ldots, \Delta_{N+M+W}\right]^{\mathrm{T}} ; \oplus$ is the binary

\footnotetext{
${ }^{2}$ The $\mathbf{S}$-degree of node $i$ is the sum of the entries of the $i$-th row in $\mathbf{S}$.
} 


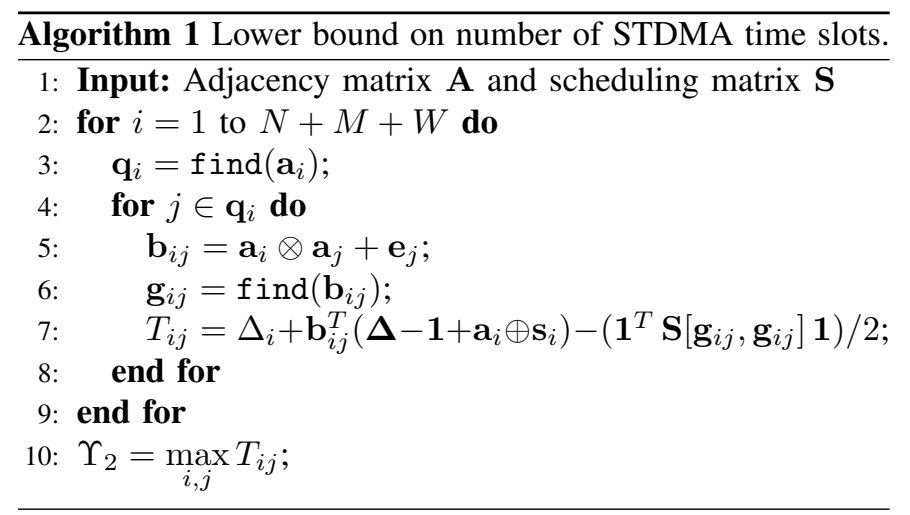

field sum; $\otimes$ is the binary field product; the find operator returns the indices to non-zero elements of the argument.

\section{RESUlts And Discussion}

\section{A. Simulation Setup}

The area under consideration is a $20 \mathrm{~m} \times 20 \mathrm{~m}$ square. Anchors are placed according to the network topology proposed in [5, Fig. 13] (properly scaled to our map size) while agents are uniformly distributed in the square. We consider a ranging standard deviation of $2 \mathrm{~cm}$ (irrespective of distance, within the confines of the map) and a TDMA time slot duration of $20 \mathrm{~ms}$. The latter considerations are based on our experimental results with the P400 UWB radios [20]. The a priori distributions of the agents' positions are Gaussian with unit variance.

We consider four different scenarios: (i) noncooperative, where agents perform TW-TOA with all anchors in communication range (i.e., $\mathcal{S}_{i}=\mathcal{N}_{i} \cap \mathcal{S}_{\text {anchors }}$ ); (ii) noncooperative selective, where agents carry out TW-TOA with at most 4 anchors (so that $\mathcal{S}_{i} \subseteq \mathcal{N}_{i} \cap \mathcal{S}_{\text {anchors }}$ ); (iii) cooperative, where agents perform TW-TOA with all nodes in communication range (i.e., $\mathcal{S}_{i}=\mathcal{N}_{i}$ ); (iv) cooperative selective, where agents acquire ranging measurements through TW-TOA with at most 4 nodes, considering that anchors in range have higher priority than neighboring agents (so that $\mathcal{S}_{i} \subseteq \mathcal{N}_{i}$ ). For scenarios (ii) and (iv), the selective ranging method is a heuristic where the main objective is to reduce the number of TW-TOA transactions for each agent. Each agent chooses randomly a node within their neighborhood and then chooses the next 3 nodes by means of a simple greedy algorithm to minimize the PEB, where neighboring anchors are chosen over neighboring agents.

We will evaluate the PEB and the upper and lower bounds on the MAC delay for each of the four scenarios and analyze the impact of the number of anchors, number of agents, the communication range, and cooperation among nodes.

\section{B. Impact of Number of Anchors}

Figs. 2 and 3 illustrate the impact on the localization accuracy and MAC delay for a clique network with 10 agents, where the number of anchors is increased from 0 to 10 . For the noncooperative scenario, the PEB decreases as anchors are added since more agent-to-anchor information is gathered by

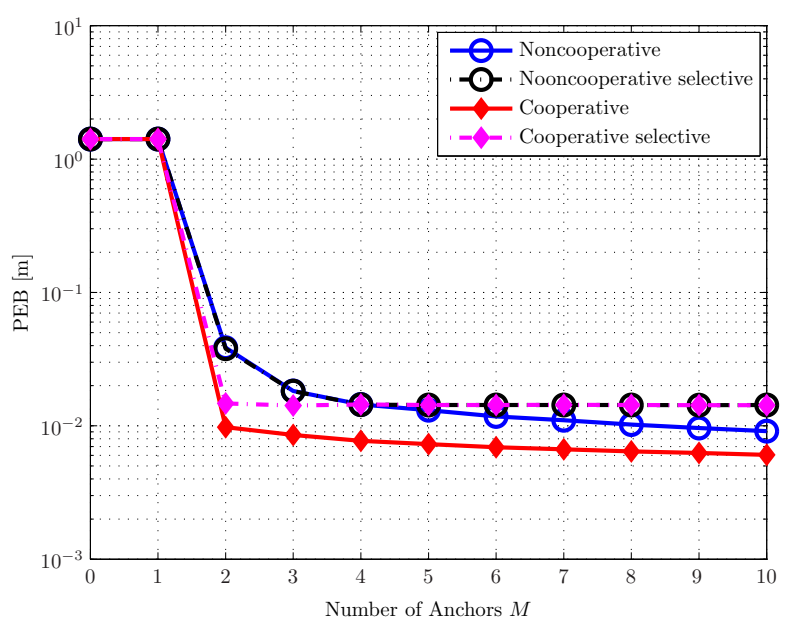

Figure 2. PEB for clique network with 10 agents and increasing number of anchors.

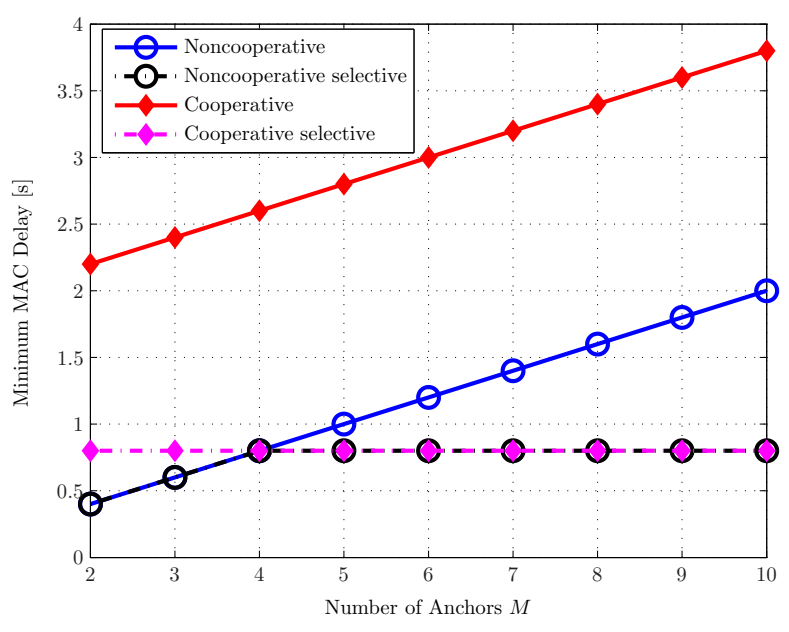

Figure 3. MAC delay for clique network with 10 agents and increasing number of anchors.

each agent. However, this induces a MAC delay that linearly increases with $M$. Given that the network is a clique, the lower and upper bounds for the MAC delay are the same, so Fig. 3 only depicts the lower bounds. For the noncooperative selective and cooperative selective scenarios, the PEB and MAC delay flatten out to around $1.5 \mathrm{~cm}$ and $0.75 \mathrm{~s}$, respectively, once agents can perform TW-TOA transactions with at least 4 nodes. Note that when having 2 or 3 anchors around, the cooperative selective case shows better position accuracy than the noncooperative one (both selective and nonselective), since an extra agent-to-agent transaction is performed by every agent. Finally, the cooperative scenario excels in terms of position accuracy, due to the collection of agent-to-agent information. However, allowing full cooperation comes with a high price in terms of MAC delay: with 10 anchors and 10 agents the MAC delay increases to $3.8 \mathrm{~s}$, which might be a constraint for highly dynamic scenarios. We conclude that cooperation is only meaningful when only a few anchors are available. 


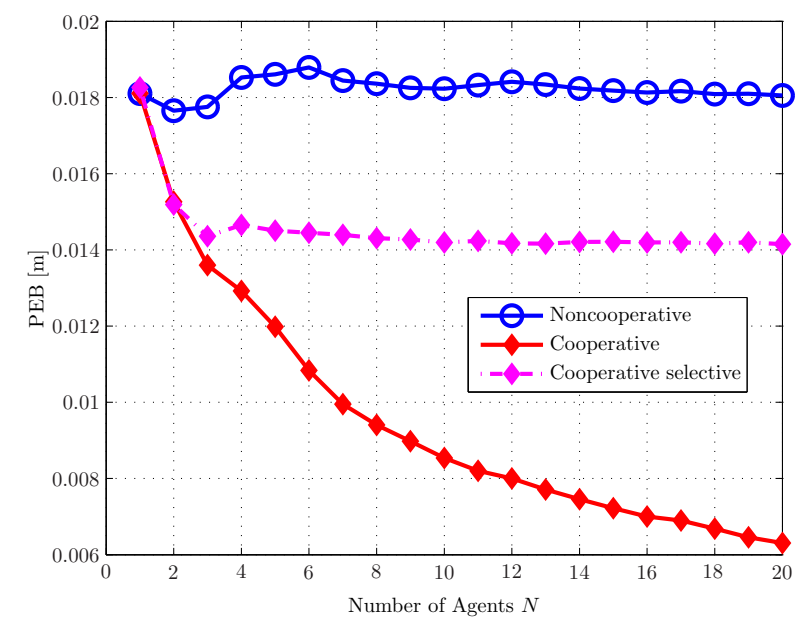

Figure 4. PEB for clique network with 3 anchors and increasing number of agents.

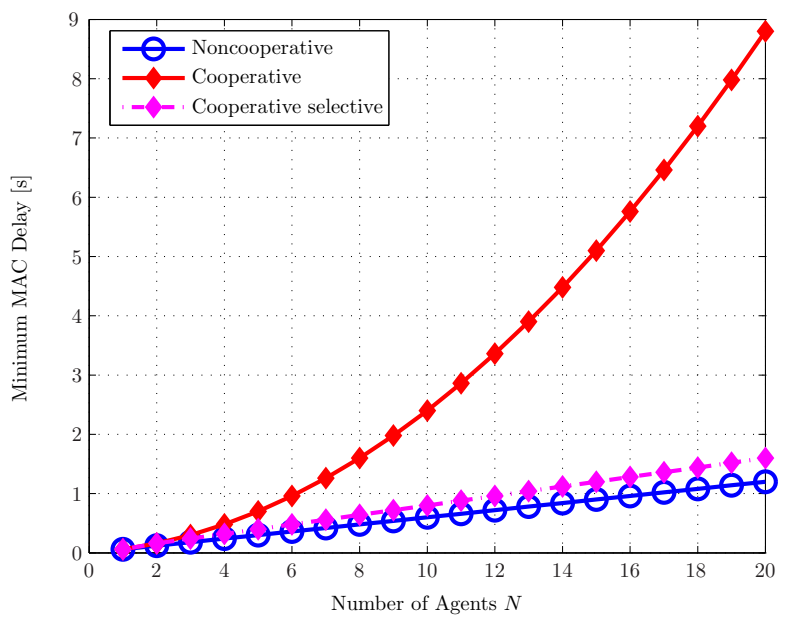

Figure 5. MAC delay for clique network with 3 anchors and increasing number of agents.

\section{Impact of Number of Agents}

Figs. 4 and 5 show the impact on the localization accuracy and the MAC delay for a clique network with 3 anchors and an increasing number of agents (from 1 to 20). Note that the noncooperative and noncooperative selective scenarios now coincide, so we do not plot the results for the selective case. Once again, the lower and upper bounds on the MAC delay are the same, and only the lower bound is shown in Fig. 5. Adding more agents to the network has no impact in the localization accuracy for the noncooperative scenarios. However, it increases the MAC delay linearly in $N$, since more agent-toanchor links need to be scheduled. The cooperative selective scenario shows a substantial performance enhancement in the position accuracy (since an extra agent-to-agent transaction per agent is being added into the network), while still maintaining a tolerable MAC delay increase (since we are only considering one extra agent-to-agent transaction per agent to complete the minimum requirement of at least ranging with 4 neighboring nodes). Regarding position accuracy, the cooperative scenario shows, once again, to outperform all other scenarios. The latter

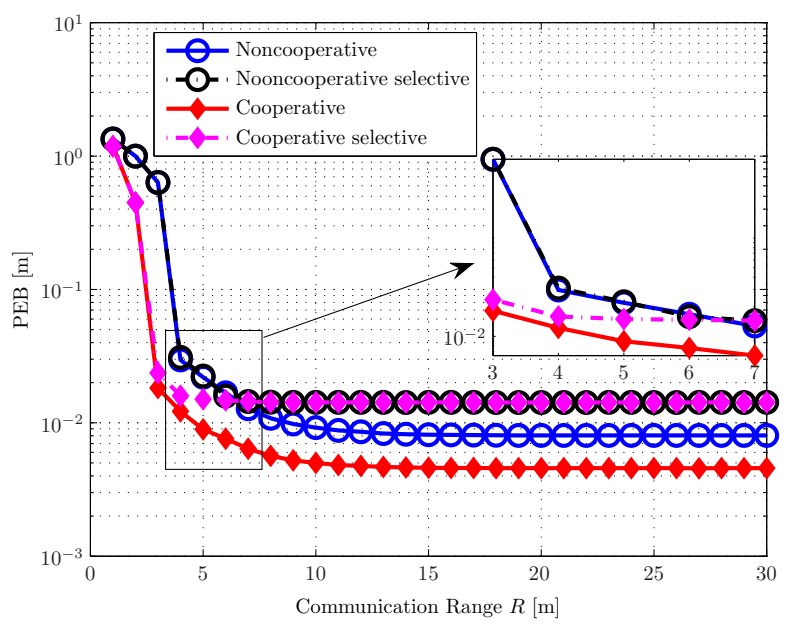

Figure 6. PEB for network with spatial reuse and increasing $R$.

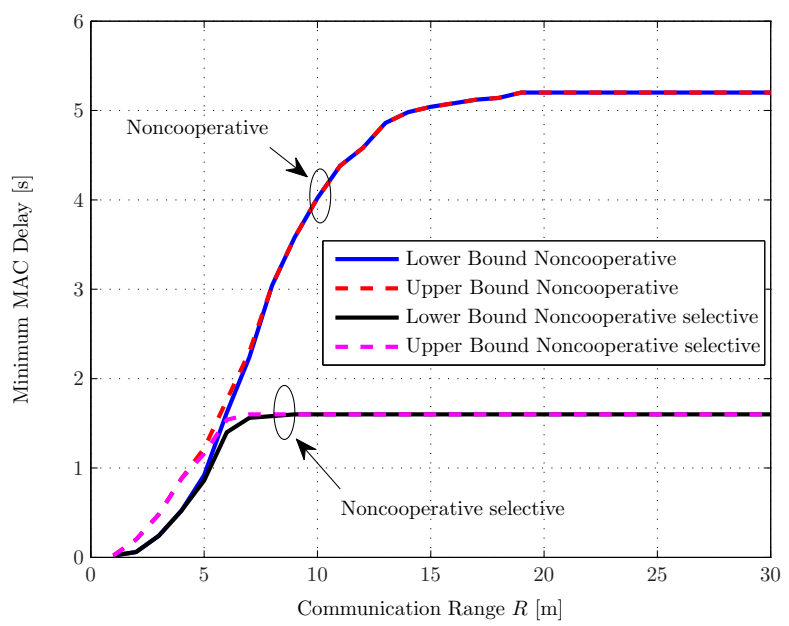

Figure 7. MAC delay for network with spatial reuse and increasing $R$ for the noncooperative scenarios.

comes with a quadratic increase in $N$ in terms of MAC delay. We conclude that when delay is a constraint, full cooperation is not feasible, and theoretical cooperative gains cannot be exploited.

\section{Impact of Communication Range}

We consider a network consisting of 20 agents and 13 anchors, and an increasing communication range $R$ from 1 $\mathrm{m}$ to $30 \mathrm{~m}$. Fig. 6 shows the influence of incrementing the communication range for the position accuracy while Figs. 8 and 7 depict the corresponding impact on the MAC delay for the cooperative and noncooperative scenarios, respectively. Similar to the increasing anchor case, the PEB reduces with increasing $R$ and remains steady once almost a fully connected network is reached at $R \approx 8 \mathrm{~m}$. The MAC delay grows fast with increasing $R$, reaching up to $N \times M \times 20 \mathrm{~ms} \approx 5 \mathrm{~s}$, and $N \times(M+(N-1)) \times 20 \mathrm{~ms} \approx 13 \mathrm{~s}$ for the noncooperative and cooperative scenarios, respectively. Evidently, the latter shows that increasing $R$ for a marginal gain in terms of accuracy can lead to large delays. The cooperative selective scenario shows to have better performance in terms of accuracy than 


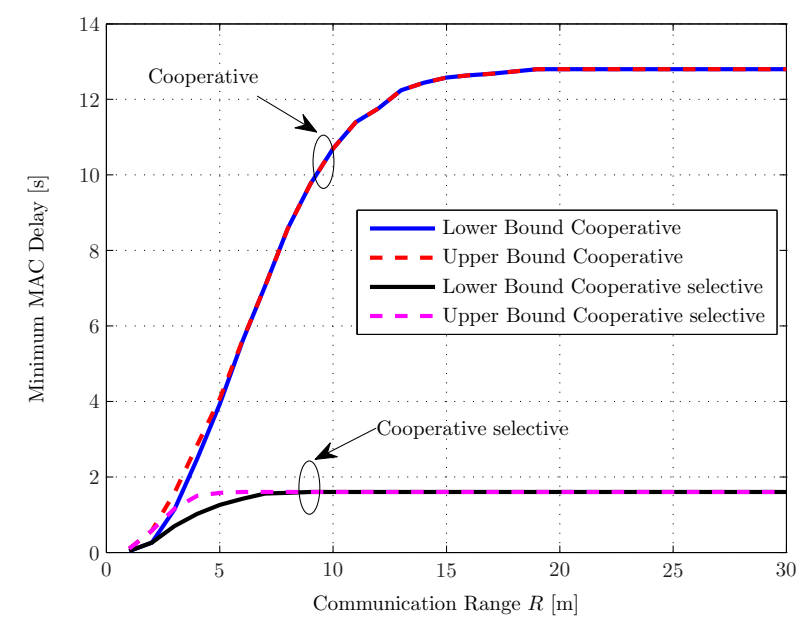

Figure 8. MAC delay for network with spatial reuse and increasing $R$ for the cooperative scenarios.

the noncooperative cases (magnified subplot in Fig. 6) with tolerable delays when the communication range is within 3.5 $\mathrm{m}$ to $6 \mathrm{~m}$. This is due to the fact that agents have fewer anchors within their neighborhood, and resort to agent-to-agent information to complete the selection of 4 neighbors. The MAC delay for the selective scenarios flatten out to around $N \times 4 \times 20 \mathrm{~ms} \approx 1.6 \mathrm{~s}$ since once a fully connected network is reached, agents perform transactions with 4 neighboring nodes.

\section{CONCLUSiOnS}

We have investigated the interplay between cooperative UWB navigation accuracy and MAC delay. For our results we considered real parameters from UWB radios obtained through experimental campaigns. We presented lower bounds on the position accuracy and the MAC delay for arbitrary finite networks. We found that traditional methods to improve accuracy, such as increasing the number of anchors or the communication range, or the implementation of cooperation among nodes comes at a cost in terms of the MAC delay. The latter has a direct impact in the update rate when dealing with mobile networks. Selective ranging has been evaluated as a possible technique to cope with this problem in order to reduce the MAC delay with reasonable position accuracy. Our results can be a guide for designers of UWB navigation systems.

Future work includes the consideration of the delay $T_{\text {comp }}$ as well as the derivation of scaling laws for both the MAC delay and the position accuracy for UWB navigation networks.

\section{ACKNOWLEDGMENT}

This research was supported in part, by the European Research Council, under Grant No. 258418 (COOPNET), and the Swedish Research Council, under Grant No. 20105889. The authors would like to thank Elad Schiller for the discussions at the initial stages of this paper.

\section{REFERENCES}

[1] K. Pahlavan and X. Li, "Indoor geolocation science and technology," IEEE Communications Magazine, vol. 40, pp. 112-118, 2002.

[2] A. F. Molisch, P. Orlik, Z. Sahinoglu, and J. Zhang, "UWB-based sensor networks and the IEEE 802.15.4a standard - a tutorial," International Conference on Communication and Networking in China, 2006.

[3] W. Hirt, "Ultra-wideband radio technology: overview and future research," Computer Communications, vol. 26, pp. 46-52, 2003.

[4] S. Gezici, Z. Tian, G. B. Giannakis, H. Kobayashi, A. F. Molisch, V. H. Poor, and Z. Sahinoglu, "Localization via ultra-wideband radios," IEEE Signal Processing Magazine, pp. 70-84, 2005.

[5] H. Wymeersch, J. Lien, and M. Z. Win, "Cooperative localization in wireless networks," Proceedings of the IEEE, vol. 97, pp. 427-450, 2009.

[6] N. Patwari, J. N. Ash, S. Kyperountas, A. O. Hero III, R. L. Moses, and N. S. Correal, "Locating the nodes," IEEE Signal Processing Magazine, vol. 22, pp. 54-59, 2005.

[7] Y. Shen and M. Z. Win, "Fundamental limits of wideband localization part I: A general framework," IEEE Transactions on Information Theory, vol. 56, pp. 4956-4980, 2010.

[8] Y. Shen, H. Wymeersch, and M. Z. Win, "Fundamental limits of wideband localization - part II: Cooperative networks," IEEE Transactions on Information Theory, vol. 56, pp. 4981-5000, 2010.

[9] I. Bucaille, A. Tonnere, L. Ouvry, and B. Denis, "MAC layer design for UWB LDR systems: PULSERS proposal," 4th Workshop on Positioning, Navigation and Communication, 2007.

[10] D. Macagnano, G. Destino, F. Esposito, and G. Abreu, "MAC performances for localization and tracking in wireless sensor networks," 4th Workshop on Positioning, Navigation and Communication, 2007.

[11] B. Denis, M. Maman, and L. Ouvry, "On the scheduling of ranging and distributed positioning updates in cooperative IR-UWB networks," International Conference on UWB, pp. 370-375, 2009.

[12] F. Sottile, A. Vesco, R. Scopigno, and M. Spirito, "MAC layer impact on the performance of real-time cooperative positioning," in Wireless Communications and Networking Conference (WCNC), 2012, pp. 18581863.

[13] T. Wang, Y. Shen, S. Mazuelas, and M. Z. Win, "Distributed scheduling for cooperative localization based on information evolution," IEEE International Conference on Communications, 2012.

[14] P. Leone and E. M. Schiller, "Self-stabilizing TDMA algorithms for dynamic wireless ad-hoc networks," in ALGOSENSORS, 2012.

[15] M. Rengasamy, E. Dutkiewicz, and M. Hedley, "MAC design and analysis for wireless sensor networks with co-operative localisation," International Symposium on Communications and Information Technologies, 2007.

[16] G. E. Garcia, L. S. Muppirisetty, and H. Wymeersch, "On the trade-off between accuracy and delay in UWB navigation," IEEE Communications Letters, vol. PP, pp. 1-4, 2012.

[17] U. Orguner and F. Gustafsson, "Target tracking with particle filters under signal propagation delays," IEEE Transactions on Signal Processing, vol. 59, pp. 2485-2495, 2011.

[18] M. Z. Win, Y. Shen, and H. Wymeersch, "On the position error bound in cooperative networks: A geometric approach," International Symposium on Spread Spectrum Techniques and Applications, pp. 637-643, 2008.

[19] C. Pedersen, T. Pedersen, and B. H. Fleury, "Exploiting network topology information to mitigate ambiguities in VMP localization," 4th IEEE International Workshop on Computational Advances in MultiSensor Adaptive Processing, pp. 57-60, 2011.

[20] "P400 data sheet," Time Domain Corp., Huntsville, AL, USA.

[21] N. Abramson, "The throughput of packet broadcasting channels," IEEE Transactions on Communications, vol. com-25, no.1, pp. 117-128, 1977.

[22] H. Van Trees, Detection, Estimation, and Modulation Theory. Wiley, 2001.

[23] W. Wang, Y. Wang, X.-Y. Li, W.-Z. Song, and O. Frieder, "Efficient interference-aware TDMA link scheduling for static wireless networks," in Proceedings of the 12th annual international conference on Mobile computing and networking, ser. MobiCom '06, 2006, pp. 262-273.

[24] I. Holyer, "The NP-completeness of edge-coloring," SIAM J. Comput., vol. 10, no. 4, pp. 718-720, 1981.

[25] R. Faudree, A. Gyárfas, R. H. Schelp, and Z. Tuza, "Induced matchings in bipartite graphs," Discrete Math., vol. 78, no. 1-2, pp. 83-87, Nov. 1989. 